\title{
Off-Line Test Selection with Test Purposes for Non-deterministic Timed Automata ${ }^{\star}$
}

\author{
Nathalie Bertrand ${ }^{1}$, Thierry Jéron ${ }^{1}$, Amélie Stainer ${ }^{1}$, and Moez Krichen ${ }^{2}$ \\ 1 INRIA Rennes - Bretagne Atlantique, Rennes, France \\ 2 Institute of Computer Science and Multimedia, Sfax, Tunisia
}

\begin{abstract}
This paper proposes novel off-line test generation techniques for non-deterministic timed automata with inputs and outputs (TAIOs) in the formal framework of the tioco conformance theory. In this context, a first problem is the determinization of TAIOs, which is necessary to foresee next enabled actions, but is in general impossible. This problem is solved here thanks to an approximate determinization using a game approach, which preserves tioco and guarantees the soundness of generated test cases. A second problem is test selection for which a precise description of timed behaviors to be tested is carried out by expressive test purposes modeled by a generalization of TAIOs. Finally, using a symbolic co-reachability analysis guided by the test purpose, test cases are generated in the form of TAIOs equipped with verdicts.
\end{abstract}

Keywords: Conformance testing, timed automata, partial observability, urgency, approximate determinization, game, test purpose.

\section{Introduction}

Conformance testing is the process of testing whether an implementation behaves correctly with respect to a specification. Implementations are considered as black boxes, i.e. the source code is unknown, only their interface with the environment is known and used to interact with the tester. In formal model-based conformance testing models are used to describe testing artifacts (specifications, implementations, test cases, ...), conformance is formally defined and test cases with verdicts are generated automatically. Then, the quality of testing may be characterized by properties of test cases which relate the verdicts of their executions with conformance (e.g. soundness). For timed models, model-based conformance testing has already been explored in the last decade, with different models and conformance relations (see e.g. [16] for a survey), and test generation algorithms (e.g. 6 14 15]). In this context, a very popular model is timed automata with inputs and outputs (TAIOs), a variant of timed automata (TAs) [1], in which observable actions are partitioned into inputs and outputs. We consider here partially observable and non-deterministic TAIOs with invariants for the modeling of urgency.

\footnotetext{
* This work was partly funded by the french ANR project Testec. An extended version of the paper with full proofs is available as a technical report 4 .
} 
One of the main difficulties encountered in test generation for TAIOs is determinization, which is impossible in general, as for TAs [1], but is required in order to foresee the next enabled actions during execution and to emit a correct verdict. Two different approaches have been taken for test generation from timed models, which induce different treatments of non-determinism. In off-line test generation test cases are first generated as TAs (or timed sequences, trees, or timed transition systems) and subsequently executed on the implementation. Test cases can then be stored and further used e.g. for regression testing and documentation. However, due to the non-determinizability of TAIOs, the approach has often been limited to deterministic or determinizable TAIOs (see e.g. [12 15]), except in 14 where the problem is solved by the use of an over-approximate determinization with fixed resources, or 8 where winning strategies of timed games are used as test cases. In on-line test generation, test cases are generated during their execution, thus can be applied to any TAIO as only possible observable actions are computed along the current finite execution, thus avoiding a complete determinization. This is of particular interest to rapidly discover errors, but may sometimes be impracticable due to a lack of reactivity (the time needed to compute successor states on-line may sometimes be incompatible with delays).

In this paper, we propose to generate test cases off-line for non-deterministic TAIOs, in the formal context of the tioco conformance theory. The determinization problem is tackled thanks to an approximate determinization with fixed resources in the spirit of [14], using a game approach [5]. Determinization is exact for known classes of determinizable TAIOs (e.g. event-clock TAs, TAs with integer resets, strongly non-Zeno TAs) if resources are sufficient. In the general case, approximate determinization guarantees soundness of generated test cases by producing a deterministic io-abstraction of the TAIO for a particular io-refinement relation, generalizing the io-refinement of [7]. Our method is more precise than [14] (see [5] for details) and preserves the richness of our model by dealing with partial observability and urgency. Behaviors of specifications to be tested are identified by means of test purposes defined as open timed automata with inputs and outputs (OTAIOs), a model generalizing TAIOs, allowing to precisely describe behaviors according to actions and clocks of the specification as well as proper clocks. Then, in the same spirit as for the TGV tool in the untimed case [1], test selection is performed by a co-reachability analysis, producing a test case in the form of a TAIO. To our knowledge, this work constitutes the most general and advanced off-line test selection approach for TAIOs.

The paper is structured as follows. In the next section we introduce the model of OTAIOs, its semantics, some notations and operations. Section 3 recalls the tioco conformance theory including expected properties relating conformance and verdicts, and an io-refinement relation preserving tioco. Section 4 presents our game approach for the approximate determinization compatible with the io-refinement. In Section 5 we detail the test selection mechanism using test purposes and prove some properties on generated test cases. 


\section{A Model of Open Timed Automata with Inputs/Outputs}

Timed automata (TAs) [1] is a usual model for time constrained systems. In the context of model-based testing, TAs have been extended to timed automata with inputs and outputs (TAIOs) whose sets of actions are partitioned into inputs, outputs and unobservable actions. In this section, we further extend TAIOs into the model of open timed automata with inputs/outputs (OTAIOs for short), by partitioning the set of clocks into proper and observed clocks. While the submodel of TAIOs (with only proper clocks) is sufficient for most testing artifacts, observed clocks of OTAIOs will be useful to express test purposes whose aim is to focus on the timed behavior of the specification. Like in 1 for TAs, we consider OTAIOs and TAIOs with location invariants to model urgency.

\subsection{Open Timed Automata with Inputs/Outputs}

We start by introducing notations and definitions concerning TAIOs and OTAIOs.

Given $X$ a finite set of clocks, and $\mathbb{R}_{\geq 0}$ the set of non-negative real numbers, a clock valuation is a mapping $v: X \rightarrow \mathbb{R}_{>0}$. If $v$ is a valuation over $X$ and $t \in \mathbb{R}$, then $v+t$ denotes the valuation which assigns to every clock $x \in X$ the value $v(x)+t$. For $X^{\prime} \subseteq X$ we write $v_{\left[X^{\prime} \leftarrow 0\right]}$ for the valuation equal to $v$ on $X \backslash X^{\prime}$ and assigning 0 to all clocks of $X^{\prime}$.

Given $M$ a non-negative integer, an $M$-bounded guard (or simply guard) over $X$ is a finite conjunction of constraints of the form $x \sim c$ where $x \in X, c \in$ $[0, M] \cap \mathbb{N}$ and $\sim \in\{<, \leq,=, \geq,>\}$. Given $g$ a guard and $v$ a valuation, we write $v \models g$ if $v$ satisfies $g$. We abuse notations and write $g$ for the set of valuations satisfying $g$. Invariants are restricted cases of guards: given $M \in \mathbb{N}$, an $M$ bounded invariant over $X$ is a finite conjunction of constraints of the form $x \triangleleft c$ where $x \in X, c \in[0, M] \cap \mathbb{N}$ and $\triangleleft \in\{<, \leq\}$. We denote by $G_{M}(X)$ (resp. $\left.I_{M}(X)\right)$ the set of $M$-bounded guards (resp. invariants) over $X$.

Definition 1 (OTAIO). An open timed automaton with inputs and outputs (OTAIO) is a tuple $\mathcal{A}=\left(L^{\mathcal{A}}, \ell_{0}^{\mathcal{A}}, \Sigma_{?}^{\mathcal{A}}, \Sigma_{!}^{\mathcal{A}}, \Sigma_{\tau}^{\mathcal{A}}, X_{p}^{\mathcal{A}}, X_{o}^{\mathcal{A}}, M^{\mathcal{A}}, I^{\mathcal{A}}, E^{\mathcal{A}}\right)$ such that:

- $L^{\mathcal{A}}$ is a finite set of locations, with $\ell_{0}^{\mathcal{A}} \in L^{\mathcal{A}}$ the initial location,

- $\Sigma_{?}^{\mathcal{A}}, \Sigma_{!}^{\mathcal{A}}$ and $\Sigma_{\tau}^{\mathcal{A}}$ are disjoint finite alphabets of input actions (noted $a ?, b ?, \ldots)$, output actions (noted $a !, b !, \ldots)$, and internal actions (noted $\left.\tau_{1}, \tau_{2}, \ldots\right)$. We note $\Sigma_{\text {obs }}^{\mathcal{A}}=\Sigma_{?}^{\mathcal{A}} \sqcup \Sigma_{!}^{\mathcal{A}}$ (where $\sqcup$ denotes the disjoint union) for the alphabet of observable actions, and $\Sigma^{\mathcal{A}}=\Sigma_{?}^{\mathcal{A}} \sqcup \Sigma_{!}^{\mathcal{A}} \sqcup \Sigma_{\tau}^{\mathcal{A}}$ for the whole set of actions.

- $X_{p}^{\mathcal{A}}$ and $X_{o}^{\mathcal{A}}$ are disjoint finite sets of proper clocks and observed clocks, respectively. We note $X^{\mathcal{A}}=X_{p}^{\mathcal{A}} \sqcup X_{o}^{\mathcal{A}}$ for the whole set of clocks.

- $M^{\mathcal{A}} \in \mathbb{N}$ is the maximal constant of $\mathcal{A}$, and we will refer to $\left(\left|X^{\mathcal{A}}\right|, M^{\mathcal{A}}\right)$ as the resources of $\mathcal{A}$, 


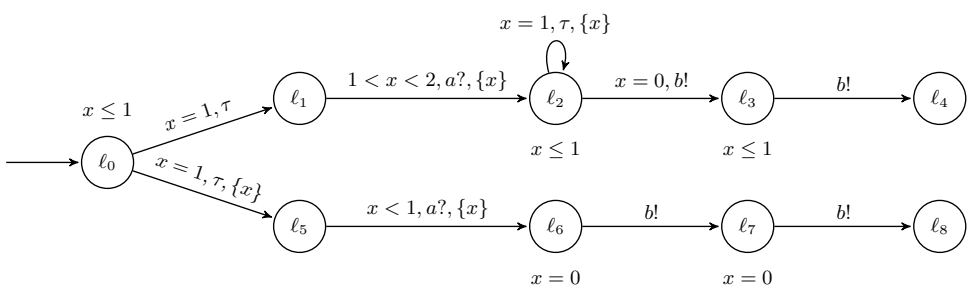

Fig. 1. Specification $\mathcal{A}$

- $I^{\mathcal{A}}: L^{\mathcal{A}} \rightarrow I_{M^{\mathcal{A}}}\left(X^{\mathcal{A}}\right)$ is a mapping labeling each location with an invariant,

$-E^{\mathcal{A}} \subseteq L^{\mathcal{A}} \times G_{M^{\mathcal{A}}}\left(X^{\mathcal{A}}\right) \times \Sigma^{\mathcal{A}} \times 2^{X_{p}^{\mathcal{A}}} \times L^{\mathcal{A}}$ is a finite set of edges where guards are defined on $X^{\mathcal{A}}$, but resets are restricted to proper clocks in $X_{p}^{\mathcal{A}}$.

The reason for introducing the OTAIO model is to have a unique model (syntax and semantics) that will be next specialized for particular testing artifacts. In particular, an OTAIO with an empty set of observed clocks $X_{o}^{\mathcal{A}}$ is a classical TAIO, and will be the model for specifications, implementations and test cases. For example, Fig. 1 represents such a TAIO for a specification $\mathcal{A}$ with clock $x$, input $a$, output $b$ and internal action $\tau$. The partition of actions reflects their roles in the testing context: the environment cannot observe internal actions, but controls inputs and observes outputs (and delays). The set of clocks is also partitioned into proper clocks, i.e. usual clocks controlled by $\mathcal{A}$, and observed clocks referring to proper clocks of another OTAIO. These cannot be reset to avoid intrusiveness, but synchronization with them in guards and invariants is allowed. In particular, test purposes have observed clocks which observe proper clocks of specifications in order to describe time constrained behaviors to be tested.

\subsection{The Semantics of OTAIOs}

The semantics of an OTAIO $\mathcal{A}=\left(L^{\mathcal{A}}, \ell_{0}^{\mathcal{A}}, \Sigma_{?}^{\mathcal{A}}, \Sigma_{!}^{\mathcal{A}}, \Sigma_{\tau}^{\mathcal{A}}, X_{p}^{\mathcal{A}}, X_{o}^{\mathcal{A}}, M^{\mathcal{A}}, I^{\mathcal{A}}, E^{\mathcal{A}}\right)$ is a timed transition system $\mathcal{T}^{\mathcal{A}}=\left(S^{\mathcal{A}}, s_{0}^{\mathcal{A}}, \Gamma^{\mathcal{A}}, \rightarrow_{\mathcal{A}}\right)$ where $S^{\mathcal{A}}=L^{\mathcal{A}} \times \mathbb{R}_{>0}^{X^{\mathcal{A}}}$ is the set of states i.e. pairs $(\ell, v)$ consisting in a location and a valuation of clocks; $s_{0}^{\mathcal{A}}=\left(\ell_{0}^{\mathcal{A}}, \overline{0}\right) \in S^{\mathcal{A}}$ is the initial state; $\Gamma^{\mathcal{A}}=\mathbb{R}_{\geq 0} \sqcup E^{\mathcal{A}} \times 2^{X_{o}^{\mathcal{A}}}$ is the set of transition labels consisting in either a delay $\delta$ or a pair $\left(e, X_{o}^{\prime}\right)$ formed by an edge and a set of observed clocks; the transition relation $\rightarrow_{\mathcal{A}} \subseteq S^{\mathcal{A}} \times \Gamma^{\mathcal{A}} \times S^{\mathcal{A}}$ is the smallest set of the following moves:

- Discrete moves: $(\ell, v) \stackrel{\left(e, X_{o}^{\prime}\right)}{\longrightarrow}\left(\ell^{\prime}, v^{\prime}\right)$ whenever there exists $e=$ $\left(\ell, g, a, X_{p}^{\prime}, \ell^{\prime}\right) \in E^{\mathcal{A}}$ such that $v \models g \wedge I^{\mathcal{A}}(\ell), X_{o}^{\prime} \subseteq X_{o}^{\mathcal{A}}$ is an arbitrary subset of observed clocks, $v^{\prime}=v_{\left[X_{p}^{\prime} \sqcup X_{o}^{\prime} \leftarrow 0\right]}$ and $v^{\prime} \models I^{\mathcal{A}}\left(\ell^{\prime}\right)$. Note that $X_{o}^{\prime}$ is unconstrained as observed clocks are controlled by another OTAIO.

- Time elapse: $(\ell, v) \stackrel{\delta}{\longrightarrow}_{\mathcal{A}}(\ell, v+\delta)$ for $\delta \in \mathbb{R}_{\geq 0}$ if $v+\delta=I^{\mathcal{A}}(\ell)$.

A partial run of $\mathcal{A}$ is a finite sequence of subsequent moves in $\left(S^{\mathcal{A}} \times \Gamma^{\mathcal{A}}\right)^{*} . S^{\mathcal{A}}$. 
For example $\rho=s_{0} \stackrel{\delta_{1}}{\longrightarrow} \mathcal{A} s_{0}^{\prime} \stackrel{\left(e_{1}, X_{o}^{1}\right)}{\longrightarrow} s_{1} \cdots s_{k-1} \stackrel{\delta_{k}}{\longrightarrow}{ }_{\mathcal{A}}^{\prime} s_{k-1}^{\prime} \stackrel{\left(e_{k}, X_{o}^{k}\right)}{\longrightarrow} s_{k}$. The sum of delays in $\rho$ is noted time $(\rho)$. A run is a partial run starting in $s_{0}^{\mathcal{A}}$. $\operatorname{Run}(\mathcal{A})$ and $\operatorname{pRun}(\mathcal{A})$ denote respectively runs and partial runs of $\mathcal{A}$. A state $s$ is reachable if there exists a run leading to $s$. A state $s$ is co-reachable from a set $S^{\prime} \subseteq S^{\mathcal{A}}$ if there is a partial run from $s$ to a state in $S^{\prime}$. We note $\operatorname{reach}(\mathcal{A})$ the set of reachable states and $\operatorname{coreach}\left(\mathcal{A}, S^{\prime}\right)$ the set of states co-reachable from $S^{\prime}$.

A (partial) sequence is a projection of a (partial) run where states are forgotten, and discrete transitions are abstracted to actions and proper resets which are grouped with observed resets. The sequence corresponding to a run $\rho=s_{0} \stackrel{\delta_{1}}{\longrightarrow} \mathcal{A}$ $s_{0}^{\prime} \stackrel{\left(e_{1}, X_{o}^{1}\right)}{\longrightarrow} s_{1} \cdots s_{k-1} \stackrel{\delta_{k}}{\longrightarrow} s_{k-1}^{\prime} \stackrel{\left(e_{k}, X_{o}^{k}\right)}{\longrightarrow} s_{k}$ is $\mu=\delta_{1} \cdot\left(a_{1}, X_{p}^{1} \sqcup X_{o}^{1}\right) \cdots \delta_{k} \cdot\left(a_{k}, X_{p}^{k} \sqcup\right.$ $\left.X_{o}^{k}\right)$ where $\forall i \in[1, k], e_{i}=\left(\ell_{i}, g_{i}, a_{i}, X_{p}^{i}, \ell_{i}^{\prime}\right)$. We then note $s_{0}^{\mathcal{A}} \stackrel{\mu}{\longrightarrow}_{\mathcal{A}} s_{k}$. We write $s_{0}^{\mathcal{A}} \stackrel{\mu}{\longrightarrow}{ }_{\mathcal{A}}$ for $\exists s_{k}, s_{0}^{\mathcal{A}} \stackrel{\mu}{\longrightarrow}{ }_{\mathcal{A}} s_{k}$. We note $\operatorname{Seq}(\mathcal{A}) \subseteq\left(\mathbb{R}_{\geq 0} \sqcup\left(\Sigma^{\mathcal{A}} \times 2^{X^{\mathcal{A}}}\right)\right)^{*}$ (respectively $\operatorname{pSeq}(\mathcal{A})$ ) the set of sequences (resp. partial sequences) of $\mathcal{A}$. For a sequence $\mu$, time $(\mu)$ denotes the sum of delays in $\mu$. For $\mu \in \operatorname{pSeq}(\mathcal{A})$, $\operatorname{Trace}(\mu) \in\left(\mathbb{R}_{\geq 0} \sqcup \Sigma_{\text {obs }}^{\mathcal{A}}\right)^{*} \cdot \mathbb{R}_{\geq 0}$ denotes the observable timed word obtained by erasing internal actions and summing delays between observable ones. It is defined inductively as follows: $\operatorname{Trace}(\varepsilon)=0, \operatorname{Trace}((\tau, X) \cdot \mu)=\operatorname{Trace}(\mu)$, $\operatorname{Trace}\left(\delta_{1} \ldots \delta_{k}\right)=\sum_{i=1}^{k} \delta_{i}$ and $\operatorname{Trace}\left(\delta_{1} \ldots \delta_{k} \cdot\left(a, X^{\prime}\right) \cdot \mu\right)=\left(\sum_{i=1}^{k} \delta_{i}\right) \cdot \operatorname{ar} \operatorname{Trace}(\mu)$ if $a \in \Sigma_{o b s}^{\mathcal{A}}$. For example Trace $\left(1 .\left(\tau, X^{1}\right) .2 .\left(a, X^{2}\right) \cdot 2 .\left(\tau, X^{3}\right)\right)=(3, a) .2$ and $\operatorname{Trace}\left(1 .\left(\tau, X^{1}\right) .2 .\left(a, X^{2}\right)\right)=(3, a) .0$. For a run $\rho$ projecting onto a sequence $\mu$, we write $\operatorname{Trace}(\rho)$ for $\operatorname{Trace}(\mu)$. The set of traces of runs of $\mathcal{A}$ is denoted by $\operatorname{Traces}(\mathcal{A}) \subseteq\left(\mathbb{R}_{\geq 0} \sqcup \Sigma_{o b s}^{\mathcal{A}}\right)^{*} \cdot \mathbb{R}_{\geq 0}$. Two OTAIOs with same sets of traces are said equivalent.

Let $\sigma \in\left(\mathbb{R}_{\geq 0} \sqcup \Sigma_{o b s}^{\mathcal{A}}\right)^{*} \cdot \mathbb{R}_{\geq 0}$ be an observable timed word, and $s \in S^{\mathcal{A}}$ a state, $\mathcal{A}$ after $\sigma=\left\{s \in S^{\mathcal{A}} \mid \exists \mu \in \operatorname{Seq}(\mathcal{A}), s_{0}^{\mathcal{A}} \stackrel{\mu}{\longrightarrow}_{\mathcal{A}} s \wedge \operatorname{trace}(\mu)=\sigma\right\}$ denotes the set of states where $\mathcal{A}$ can stay after observing the trace $\sigma$. We note elapse $(s)=$ $\left\{t \in \mathbb{R}_{\geq 0} \mid s \stackrel{t}{\longrightarrow} \mathcal{A}\right\}$ the set of possible delays in $s$, and $\operatorname{out}(s)=\left\{a \in \Sigma_{!}^{\mathcal{A}} \mid\right.$ $\left.\exists X \subseteq X^{\mathcal{A}}, s \stackrel{(a, X)}{\longrightarrow}{ }_{\mathcal{A}}\right\} \sqcup \operatorname{elapse}(s)$ (and $i n(s)=\left\{a \in \Sigma_{?}^{\mathcal{A}} \mid s \stackrel{(a, X)}{\longrightarrow}_{\mathcal{A}}\right\}$ ) for the set of outputs and delays (respectively inputs) that can be observed from $s$. For $S^{\prime} \subset S^{\mathcal{A}}, \operatorname{out}\left(S^{\prime}\right)=\bigcup_{s \in S^{\prime}} \operatorname{out}(s)$ and $\operatorname{in}\left(S^{\prime}\right)=\bigcup_{s \in S^{\prime}}$ in $(s)$.

\subsection{Properties and Operations}

An TAIO $\mathcal{A}$ is deterministic (and called a DTAIO) whenever for any $\sigma \in$ $\operatorname{Traces}(\mathcal{A}), s_{0}^{\mathcal{A}}$ after $\sigma$ is a singleton 1 . A TAIO $\mathcal{A}$ is determinizable if there exists an equivalent DTAIO. It is well-known that some TAs are not determinizable [1; moreover, the determinizability of TAs is an undecidable problem, even with fixed resources 18,10 .

An OTAIO $\mathcal{A}$ is complete if in any location $\ell, I^{\mathcal{A}}(\ell)=$ true, for any action $a \in \Sigma^{\mathcal{A}}$, the disjunction of guards of transitions leaving $\ell$ and labeled by $a$ is true.

\footnotetext{
${ }^{1}$ The notion of determinism is needed here and defined only for TAIOs. For OTAIOs the right definition would consider the projection of $s_{0}^{\mathcal{A}}$ after $\sigma$ which forgets values of observed clocks, as these introduce "environmental" non-determinism.
} 
This entails $\operatorname{Traces}(\mathcal{A})=\left(\Sigma^{\mathcal{A}}\right)^{*}$ (the universal language). $\mathcal{A}$ is input-complete in $s \in \operatorname{reach}(\mathcal{A})$, if $\forall a \in \Sigma_{?}^{\mathcal{A}}, s \stackrel{a}{\rightarrow}$. $\mathcal{A}$ is non-blocking if $\forall s \in \operatorname{reach}(\mathcal{A}), \forall t \in$ $\left.\mathbb{R}_{\geq 0}, \exists \mu \in \operatorname{pSeq}(\mathcal{A}) \cap\left(\mathbb{R}_{\geq 0} \sqcup\left(\Sigma_{!}^{\mathcal{A}} \sqcup \Sigma_{\tau}^{\mathcal{A}}\right) \times 2^{X^{\mathcal{A}}}\right)\right)^{*}$, time $(\mu)=t \wedge s \stackrel{\mu}{\rightarrow}$.

We now define a product operation on OTAIOs which extends the classical product of TAs, with a particular attention to observed clocks:

Definition 2 (Product). The product of two OTAIOs with same alphabets $\mathcal{A}^{i}=\left(L^{i}, \ell_{0}^{i}, \Sigma_{?}, \Sigma_{!}, \Sigma_{\tau}, X_{p}^{i}, X_{o}^{i}, M^{i}, I^{i}, E^{i}\right), i=1,2$, and disjoint sets of proper clocks $\left(X_{p}^{1} \cap X_{p}^{2}=\emptyset\right)$ is the OTAIO $\mathcal{A}^{1} \times \mathcal{A}^{2}=\left(L, \ell_{0}, \Sigma_{?}, \Sigma_{!}, \Sigma_{\tau}, X_{p}, X_{o}, M, I, E\right)$ where: $L=L^{1} \times L^{2} ; \ell_{0}=\left(\ell_{0}^{1}, \ell_{0}^{2}\right) ; X_{p}=X_{p}^{1} \sqcup X_{p}^{2}, X_{o}=\left(X_{o}^{1} \cup X_{o}^{2}\right) \backslash X_{p}$; $M=\max \left(M^{1}, M^{2}\right) ; \forall\left(\ell^{1}, \ell^{2}\right) \in L, I\left(\left(\ell^{1}, \ell^{2}\right)\right)=I^{1}\left(\ell^{1}\right) \wedge I^{2}\left(\ell^{2}\right) ;$ and $\left(\left(\ell^{1}, \ell^{2}\right), g^{1} \wedge g^{2}, a, X_{p}^{\prime 1} \sqcup X_{p}^{\prime 2},\left(\ell^{\prime 1}, \ell^{\prime 2}\right)\right) \in E$ if $\left(\ell^{i}, g^{i}, a, X_{p}^{\prime i}, \ell^{\prime i}\right) \in E^{i}, i=1,2$.

Intuitively, $\mathcal{A}^{1}$ and $\mathcal{A}^{2}$ synchronize on both time and common actions (including internal ones). $\mathcal{A}^{2}$ may observe proper clocks of $\mathcal{A}^{1}$ with its observed clocks $X_{o}^{1} \cap X_{p}^{2}$, and vice versa. The set of proper clocks of $\mathcal{A}^{1} \times A^{2}$ is the union of proper clocks of $\mathcal{A}^{1}$ and $A^{2}$, and observed clocks are those observed clocks of one OTAIO that are not proper. For example, the OTAIO in Fig. 3 represents the product of the TAIO $\mathcal{A}$ in Fig. 1 and the OTAIO $\mathcal{T} \mathcal{P}$ of Fig. 2

The product is the right operation for intersecting sets of sequences. In fact, let $\mathcal{A}^{1} \uparrow^{\left(X_{p}^{2}, X_{o}^{2}\right)}$ (respectively $\mathcal{A}^{2} \uparrow\left(X_{p}^{1}, X_{o}^{1}\right)$ ) denote the same TAIO $\mathcal{A}^{1}$ (resp. $\mathcal{A}^{2}$ ) defined on $\left(X_{p}^{1}, X_{p}^{2} \cup X_{o}^{2} \cup X_{o}^{1} \backslash X_{p}^{1}\right)$ (resp. on $\left(X_{p}^{2}, X_{p}^{1} \cup X_{o}^{2} \cup X_{o}^{2} \backslash X_{p}^{2}\right)$ ). Then we get: $\operatorname{Seq}\left(\mathcal{A}^{1} \times \mathcal{A}^{2}\right)=\operatorname{Seq}\left(\mathcal{A}^{1 \uparrow}\left(X_{p}^{2}, X_{o}^{2}\right)\right) \cap \operatorname{Seq}\left(\mathcal{A}^{2 \uparrow}\left(X_{p}^{1}, X_{o}^{1}\right)\right)$.

An OTAIO equipped with a set of states $F \subseteq S^{\mathcal{A}}$ can play the role of an acceptor. $\operatorname{Run}_{F}(\mathcal{A})$ denotes the set of runs accepted in $F$, those runs ending in $F, \operatorname{Seq}_{F}(\mathcal{A})$ denotes the set of sequences of accepted runs and $\operatorname{Traces}_{F}(\mathcal{A})$ the set of their traces. By abuse of notation, if $L$ is a subset of locations $L^{\mathcal{A}}$, we write $\operatorname{Run}_{L}(\mathcal{A})$ for $\operatorname{Run}_{L \times \mathbb{R}_{>0}^{X} \mathcal{A}}(\mathcal{A})$ and similarly for $\operatorname{Seq}_{L}(\mathcal{A})$ and $\operatorname{Traces}_{L}(\mathcal{A})$. Note that for the product $\mathcal{A}^{1} \times \mathcal{A}^{2}$, if $F^{1}$ and $F^{2}$ are subsets of states of $\mathcal{A}^{1}$ and $\mathcal{A}^{2}$ respectively, we get: $\operatorname{Seq}_{F^{1} \times F^{2}}\left(\mathcal{A}^{1} \times \mathcal{A}^{2}\right)=\operatorname{Seq}_{F^{1}}\left(\mathcal{A}^{1 \uparrow} X_{p}^{2}, X_{o}^{2}\right) \cap \operatorname{Seq}_{F^{2}}\left(\mathcal{A}^{2 \uparrow} X_{p}^{1}, X_{o}^{1}\right)$.

\section{Conformance Testing Theory}

In this section, we recall the conformance relation tioco [14, that formally defines the set of correct implementations of a given TAIO specification. We then define test cases, formalize their executions, verdicts and expected properties. Finally, we introduce a refinement relation between TAIOs that preserves tioco.

\subsection{The tioco Conformance Theory}

We consider that the specification is given as a (possibly non-deterministic) TAIO $\mathcal{A}=\left(L^{\mathcal{A}}, \ell_{0}^{\mathcal{A}}, \Sigma_{?}, \Sigma_{!}, \Sigma_{\tau}, X_{p}^{\mathcal{A}}, \emptyset, M^{\mathcal{A}}, I^{\mathcal{A}}, E^{\mathcal{A}}\right)$. The implementation is a black box, unknown except for its alphabet of observable actions, which is the same as the one of $\mathcal{A}$. As usual, in order to formally reason about conformance, we assume that the implementation can be modeled by an (unknown) TAIO $\mathcal{I}=\left(L^{\mathcal{I}}, \ell_{0}^{\mathcal{I}}, \Sigma_{?}, \Sigma_{!}, \Sigma_{\tau}^{\mathcal{I}}, X_{p}^{\mathcal{I}}, \emptyset, M^{\mathcal{I}}, I^{\mathcal{I}}, E^{\mathcal{I}}\right)$ with same observable alphabet as $\mathcal{A}$, 
and require that it is input-complete and non-blocking. The set of such possible implementations of $\mathcal{A}$ is denoted by $\mathcal{I}(\mathcal{A})$. Among these, the conformance relation tioco [14 formally defines which ones conform to $\mathcal{A}$, naturaly extending the ioco relation of Tretmans [17] to timed systems:

Definition 3 (Conformance relation). Let $\mathcal{A}$ be a TAIO and $\mathcal{I} \in \mathcal{I}(\mathcal{A})$, $\mathcal{I}$ tioco $\mathcal{A}$ if $\forall \sigma \in \operatorname{Traces}(\mathcal{A}), \operatorname{out}(\mathcal{I}$ after $\sigma) \subseteq \operatorname{out}(\mathcal{A}$ after $\sigma)$.

Intuitively, $\mathcal{I}$ conforms to $\mathcal{A}(\mathcal{I}$ tioco $\mathcal{A})$ if after any timed trace enabled in $\mathcal{A}$, every output or delay of $\mathcal{I}$ is specified in $\mathcal{A}$. In practice, conformance is checked by test cases run on implementations. In our setting, we define test cases as deterministic TAIOs equipped with verdicts defined by a partition of states.

Definition 4 (Test case, test suite). Given a specification TAIO $\mathcal{A}$, a test case for $\mathcal{A}$ is a pair $(\mathcal{T C}$, Verdicts) consisting of a deterministic TAIO (DTAIO) $\mathcal{T C}=\left(L^{\mathcal{T C}}, \ell_{0}^{\mathcal{T} C}, \Sigma_{?}^{\mathcal{T} C}, \Sigma_{!}^{\mathcal{T} C}, \Sigma_{\tau}^{\mathcal{T} C}, X_{p}^{\mathcal{T} C}, \emptyset, M^{\mathcal{T} C}, I^{\mathcal{T} C}, E^{\mathcal{T} C}\right)$ together with a partition Verdicts of the set of states $S^{\mathcal{T C}}=$ None $\sqcup$ Inconc $\sqcup$ Pass $\sqcup$ Fail. States outside None are called verdict states. We require that $\Sigma_{?}^{\mathcal{T} C}=\Sigma_{!}^{\mathcal{A}}$ and $\Sigma_{!}^{\mathcal{T C}}=\Sigma_{?}^{\mathcal{A}}$, $I^{\mathcal{T C}}(\ell)=$ true for all $\ell \in L^{\mathcal{T C}}$, and $\mathcal{T C}$ is input-complete in all None states, meaning that it is ready to receive any input from the implementation before reaching a verdict. A test suite is a set of test cases.

The verdict of an execution $\sigma \in \operatorname{Traces}(\mathcal{T C})$, noted $\operatorname{Verdict}(\sigma, \mathcal{T C})$, is Pass, Fail, Inconc or None if $\mathcal{T C}$ after $\sigma$ is included in the corresponding states set. We note $\mathcal{I}$ fails $T C$ if some execution $\sigma$ of $\mathcal{T C} \| \mathcal{I}$ leads $\mathcal{T C}$ to a Fail state, i.e. when $\operatorname{Traces}{ }_{\text {Fail }}(\mathcal{T C}) \cap \operatorname{Traces}(\mathcal{I}) \neq \emptyset \square$. Notice that this is only a possibility to reach the Fail verdict among the infinite set of executions.

We now introduce soundness, a crucial property ensured by our test generation method and strictness that will be ensured when determinization is exact.

Definition 5 (Test case properties). A test suite $\mathcal{T} \mathcal{S}$ for $\mathcal{A}$ is sound if no conformant implementation is rejected by the test suite i.e. $\forall \mathcal{I} \in \mathcal{I}(\mathcal{A}), \forall \mathcal{T C} \in$ $\mathcal{T S}, \mathcal{I}$ fails $\mathcal{T C} \Rightarrow \neg(\mathcal{I}$ tioco $\mathcal{A})$. It is strict if non-conformance is detected as soon as it occurs i.e. $\forall \mathcal{I} \in \mathcal{I}(A), \forall \mathcal{T C} \in \mathcal{T} \mathcal{S}, \neg(\mathcal{I} \| \mathcal{T C}$ tioco $\mathcal{A}) \Rightarrow \mathcal{I}$ fails $\mathcal{T C}$.

\subsection{Refinement Preserving tioco}

We introduce an io-refinement relation between TAIOs, a generalization to nondeterministic TAIOs of the io-refinement between DTAIOs introduced in [7], itself a generalization of alternating simulation [2. We prove that io-abstraction (the inverse relation) preserves tioco: if $\mathcal{I}$ conforms to $\mathcal{A}$, it also conforms to any io-abstraction $\mathcal{B}$ of $\mathcal{A}$. This will ensure that soundness of test cases is preserved by the approximate determinization defined in Section 4.

\footnotetext{
$\overline{2}$ The execution of a test case $\mathcal{T C}$ on an implementation $\mathcal{I}$ is usually modeled by the standard parallel composition $\mathcal{T C} \| \mathcal{I}$. Due to space limitations, $\|$ is not defined here, but we use its trace properties: $\operatorname{Traces}(\mathcal{I} \| \mathcal{T} \mathcal{C})=\operatorname{Traces}(\mathcal{I}) \cap \operatorname{Traces}(\mathcal{T C})$.
} 
Definition 6. Let $\mathcal{A}$ and $\mathcal{B}$ be two TAIOs with same input and output alphabets, we say that $\mathcal{A}$ io-refines $\mathcal{B}$ (or $\mathcal{B}$ io-abstracts $\mathcal{A}$ ) and note $\mathcal{A} \preceq \mathcal{B}$ if

(i) $\forall \sigma \in \operatorname{Traces}(\mathcal{B})$, out $(\mathcal{A}$ after $\sigma) \subseteq \operatorname{out}(\mathcal{B}$ after $\sigma)$ and

(ii) $\forall \sigma \in \operatorname{Traces}(\mathcal{A})$, in $(\mathcal{B}$ after $\sigma) \subseteq \operatorname{in}(\mathcal{A}$ after $\sigma)$.

It can be proved that $\preceq$ is a preorder relation. Moreover, as $(i i)$ is always satisfied if $\mathcal{A}$ is input-complete, for $\mathcal{I} \in \mathcal{I}(\mathcal{A}), \mathcal{I}$ tioco $\mathcal{A}$ is equivalent to $\mathcal{I} \preceq \mathcal{A}$. Вy transitivity of $\preceq$, Proposition 1 states that io-refinement preserves conformance. Its Corollary 1 says that io-abstraction preserves soundness of test suites and will later justify that if a TAIO $\mathcal{B}$ io-abstracting $\mathcal{A}$ is obtained by approximate determinization, a sound test suite generated from $\mathcal{B}$ is still sound for $\mathcal{A}$.

Proposition 1. If $\mathcal{A} \preceq \mathcal{B}$ then $\forall \mathcal{I} \in \mathcal{I}(\mathcal{A})(=\mathcal{I}(\mathcal{B})), \mathcal{I}$ tioco $\mathcal{A} \Rightarrow \mathcal{I}$ tioco $\mathcal{B}$.

Corollary 1. If $A \preceq B$ then any sound test suite for $\mathcal{B}$ is also sound for $\mathcal{A}$.

\section{Approximate Determinization Preserving tioco}

We recently proposed a game approach to determinize or provide a deterministic over-approximation for TAs [5]. Determinization is exact on all known classes of determinizable TAIOs (e.g. event-clock TAs, TAs with integer resets, strongly non-Zeno TAs) if resources are sufficient. Provided a couple of extensions, this method can be adapted to the context of testing for building a deterministic ioabstraction of a given TAIO. Thanks to Proposition 1 the construction preserves tioco, and Corollary 1 guarantees the soundness of generated test cases.

The approximate determinization uses the classical region construction [1. As for classical TAs, the regions form a partition of valuations over a given set of clocks which allows to make abstractions and decide properties like the reachability of a location. We note $\operatorname{Reg}_{(X, M)}$ the set of regions over clocks $X$ with maximal constant $M$. A region $r^{\prime}$ is a time-successor of a region $r$ if $\exists v \in$ $r, \exists t \in \mathbb{R}_{\geq 0}, v+t \in r^{\prime}$. Given $X$ and $Y$ two finite sets of clocks, a relation between clocks of $X$ and $Y$ is a finite conjunction $C$ of atomic constraints of the form $x-y \sim c$ where $x \in X, y \in Y, \sim \in\{<,=,>\}$ and $c \in \mathbb{N}$. When $c \in\left[-M^{\prime}, M\right]$, for $M, M^{\prime} \in \mathbb{N}, \operatorname{Rel}_{M, M^{\prime}}(X, Y)$ we denote the set of relations between $X$ and $Y$.

\subsection{A Game Approach to Determinize Timed Automata}

The technique presented in [5] applies first to TAs, i.e. the alphabet only consists of one kind of actions (output actions), and the invariants are all trivial. Given such a TA $\mathcal{A}$ over the set of clocks $X^{\mathcal{A}}$, the goal is to build a deterministic TA $\mathcal{B}$ with $\operatorname{Traces}(\mathcal{A})=\operatorname{Traces}(\mathcal{B})$ as often as possible, or $\operatorname{Traces}(\mathcal{A}) \subseteq \operatorname{Traces}(\mathcal{B})$. In order to do so, resources of $\mathcal{B}$ (number of clocks $k$ and maximal constant $M^{\mathcal{B}}$ ) are fixed, and a finite 2-player turn-based safety game $\mathcal{G}_{\mathcal{A},\left(k, M^{\mathcal{B}}\right)}$ is built. The two players, Spoiler and Determinizator, alternate moves, the objective of player Determinizator being to remain in a set of safe states where intuitively, for sure no over-approximation has been performed. Every strategy for Determinizator yields a deterministic automaton $\mathcal{B}$ with $\operatorname{Traces}(\mathcal{A}) \subseteq \operatorname{Traces}(\mathcal{B})$, and 
every winning strategy induces a deterministic TA $\mathcal{B}$ equivalent to $\mathcal{A}$. It is well known that for this kind of games, winning strategies can be chosen positional and computed in linear time in the size of the arena.

Let us now give more details on the definition of the game. Let $X^{\mathcal{B}}$ be a set of clocks of cardinality $k$. The initial state of the game is a state of Spoiler consisting of the initial location of $\mathcal{A}$, the simplest relation between $X^{\mathcal{A}}$ and $X^{\mathcal{B}}: \forall x \in$ $X^{\mathcal{A}}, \forall y \in X^{\mathcal{B}}, x-y=0$, a marking $\top$ indicating that no over-approximation was done so far, together with the null region over $X^{\mathcal{B}}$. In each of his states, Spoiler challenges Determinizator by proposing a region $r \in \operatorname{Reg}_{\left(X^{\mathcal{B}}, M^{\mathcal{B}}\right)}$, and an action $a \in \Sigma$. Determinizator answers by deciding the subset of clocks $Y^{\prime} \subseteq X^{\mathcal{B}}$ he wishes to reset. The next state of Spoiler contains a region over $X^{\mathcal{B}}\left(r^{\prime}=\right.$ $\left.r_{\left[Y^{\prime} \leftarrow 0\right]}\right)$, and a finite set of configurations: triples formed of a location of $\mathcal{A}$, a relation between clocks in $X^{\mathcal{A}}$ and clocks in $X^{\mathcal{B}}$, and a boolean marking ( $\top$ or $\perp$ ). A state of Spoiler thus constitutes a states estimate of $\mathcal{A}$, and the role of the markings is to indicate whether over-approximations possibly happened. Bad states Determinizator wants to avoid are states where all configurations are marked $\perp$, i.e. configurations where an approximation possibly happened.

A strategy for Determinizator thus assigns to each state of Determinizator a set $Y^{\prime} \subseteq X^{\mathcal{B}}$ of clocks to be reset. With every strategy for Determinizator $\Pi$ we associate the TA $\mathcal{B}=\operatorname{Aut}(\Pi)$ obtained by merging a transition of Spoiler with the transition chosen by Determinizator just after. The following theorem links strategies of Determinizator with deterministic over-approximations of the original traces language and enlightens the interest of the game:

Theorem 1 ([5]). Let $\mathcal{A}$ be a $T A, k, M^{\mathcal{B}} \in \mathbb{N}$. For any strategy $\Pi$ of Determinizator in $\mathcal{G}_{\mathcal{A},\left(k, M^{\mathcal{B}}\right)}, \mathcal{B}=\operatorname{Aut}(\Pi)$ is a deterministic TA over resources $\left(k, M^{\mathcal{B}}\right)$ with $\operatorname{Traces}(\mathcal{A}) \subseteq \operatorname{Traces}(\mathcal{B})$. Moreover, if $\Pi$ is winning, $\operatorname{Traces}(\mathcal{A})=\operatorname{Traces}(\mathcal{B})$.

\subsection{Extensions to TAIOs and Adaptation to tioco}

In the context of model-based testing, the above-mentioned determinization technique must be adapted to TAIOs, as detailed in [5], and summarized below. First the model of TAIOs is more expressive than TAs, incorporating internal actions and invariants. Second, inputs and outputs must be treated differently in order to build from a TAIO $\mathcal{A}$ a DTAIO $\mathcal{B}$ such that $\mathcal{A} \preceq \mathcal{B}$ and then preserve tioco.

Internal actions: Specifications naturally include internal actions that cannot be observed during test executions, and should thus be removed during determinization. In order to do so, a closure by internal actions is performed for each state during the construction of the game. To this attempt, states of the game have to be extended since internal actions might be enabled only from some time-successor of the region associated with the state. Therefore, each configuration is associated with a proper region which is a time-successor of the initial region of the state. The closure by silent transitions is effectively computed the same way as successors in the original construction when Determinizator does not reset any clock, computations thus terminate for the same reasons. It is well 
known that TAs with silent transitions are strictly more expressive than standard TAs [3]. Therefore, our approximation can be coarse, but it performs as well as possible with its available clock information.

Invariants: Modeling urgency is quite important and using invariants to this aim is classical. Without the ability to express urgency, for instance, any inactive system would conform to all specifications. Ignoring all invariants in the approximation surely yields an io-abstraction: delays (considered as outputs) are over-approximated. In order to be more precise while preserving $\preceq$, with each state of the game is associated the most restrictive invariant containing invariants of all the configurations in the state. In the computation of the successors, invariants are treated as guards and their validity is verified at both extremities of the transition. A state whose invariant is strictly over-approximated is unsafe.

io-abstraction vs. over-approximation: Rather than over-approximating a given TAIO $\mathcal{A}$, we aim here at building a DTAIO $\mathcal{B}$ io-abstracting $\mathcal{A}(\mathcal{A} \preceq \mathcal{B})$. Successors by output are over-approximated as in the original game, while successors by inputs must be under-approximated. The over-approximated closure by silent transitions is not suitable to under-approximation. Therefore, states of the game are extended to contain both over- and under-approximated closures. Thus, the unsafe successors by an input are not built.

All in all, these modifications allow to deal with the full TAIO model with invariants, silent transitions and inputs/outputs, consistently with the io-abstraction. Fig 4 represents a part of this game for the TAIO of Fig. 3 . The new game then enjoys the following nice property:

Proposition 2 ([5] $\left.]_{3}^{3}\right)$. Let $\mathcal{A}$ be a TAIO, $k, M^{\mathcal{B}} \in \mathbb{N}$. For any strategy $\Pi$ of Determinizator in $\mathcal{G}_{\mathcal{A},\left(k, M^{\mathcal{B}}\right)}, \mathcal{B}=\operatorname{Aut}(\Pi)$ is a DTAIO over resources $\left(k, M^{\mathcal{B}}\right)$ with $\mathcal{A} \preceq \mathcal{B}$. Moreover, if $\Pi$ is winning, $\operatorname{Traces}(\mathcal{A})=\operatorname{Traces}(\mathcal{B})$.

In other words, the approximations produced by our method are deterministic io-abstractions of the initial specification, hence our approach preserves tioco (Proposition 1) and soundness of test cases (Corollary 1). In comparison, the algorithm proposed in [14 is an over-approximation, thus preserves tioco only if the specification is input-complete. Moreover it does not preserve urgency.

\section{Off-Line Test Case Generation}

In this section we first define test purposes and then give the principles for off-line test selection with test purposes and properties of generated test cases.

\subsection{Test Purposes}

Test purposes are practical means to select behaviors to be tested, either focusing on usual behaviors, or on suspected errors in implementations. In this work we choose the following definition, and discuss alternatives in the conclusion.

\footnotetext{
${ }^{3}$ Note that the proof of this proposition in [5] considers a stronger refinement relation, thus implies the same result for the present refinement relation.
} 
Definition 7 (Test purpose). For a specification TAIO $\mathcal{A}$, a test purpose is a $\operatorname{pair}(\mathcal{T} \mathcal{P}$, Accept $)$ where $\mathcal{T} \mathcal{P}=\left(L^{\mathcal{T} P}, \ell_{0}^{\mathcal{T} P}, \Sigma_{?}, \Sigma_{!}, \Sigma_{\tau}, X_{p}^{\mathcal{T} P}, X_{o}^{\mathcal{T} P}, M^{\mathcal{T} P}, I^{\mathcal{T} P}, E^{\mathcal{T} P}\right)$ is a complete OTAIO (in particular $\forall \ell \in L^{\mathcal{T} P}, I^{\mathcal{T} P}(\ell)=$ true ) with $X_{o}^{\mathcal{T} P}=X_{p}^{\mathcal{A}}$ (TP observes proper clocks of $\mathcal{A})$, and Accept $\subseteq L^{\mathcal{T} P}$ is a subset of trap locations.

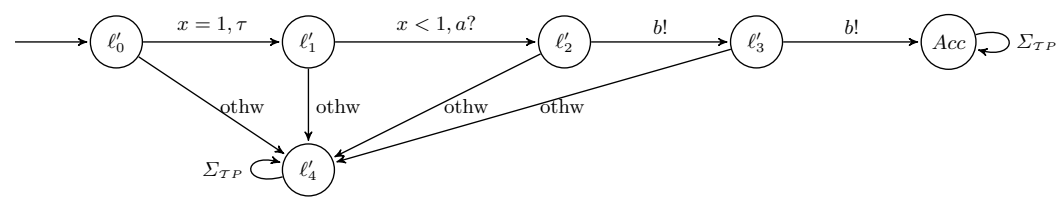

Fig. 2. Test purpose $\mathcal{T} \mathcal{P}$

Fig. 2 represents a test purpose for the specification $\mathcal{A}$ of Fig. 1, It has no proper clock and observes the unique clock $x$ of $\mathcal{A}$. It accepts sequences where $\tau$ occurs at $x=1$, followed by an input $a$ ? at $x<1$ (thus focusing on the lower branch of $\mathcal{A}$ where $x$ is reset), and two subsequent $b$ !'s. The label othw (for otherwise) is an abbreviation for the complement of specified transitions.

\subsection{Principle of Test Generation}

Given a specification TAIO $\mathcal{A}$ and a test purpose $\left(\mathcal{T} \mathcal{P}, \operatorname{Accept}^{\mathcal{T} P}\right)$, the aim is to build a sound and, if possible strict test case $(\mathcal{T C}$, Verdicts $)$. It should also deliver Pass verdicts on traces of sequences of $\mathcal{A}$ accepted by $\mathcal{T} \mathcal{P}$, as formalized by the following property:

Definition 8. A test suite $\mathcal{T S}$ for $\mathcal{A}$ and $\mathcal{T} \mathcal{P}$ is precise if $\forall \mathcal{T C} \in \mathcal{T S}, \forall \sigma \in$ $\left(\Sigma_{\text {obs }}^{\mathcal{A}}\right)^{*}, \operatorname{Verdict}(\sigma, \mathcal{T} \mathcal{C})=\operatorname{Pass} \Longleftrightarrow \sigma \in \operatorname{Traces}\left(\operatorname{Seq}_{\text {Accept }}^{\mathcal{T} P}(\mathcal{T} \mathcal{P}) \cap \operatorname{Seq}(\mathcal{A})\right)$.

The different steps of test generation are described in the following paragraphs.

Product: we first build the TAIO $\mathcal{P}=\mathcal{A} \times \mathcal{T} \mathcal{P}$ associated with the set of marked locations Accept $^{\mathcal{P}}=L^{\mathcal{A}} \times$ Accept $^{\mathcal{T} P}$. Fig. 3 represents this product $\mathcal{P}$ for the specification $\mathcal{A}$ in Fig. 1 and the test purpose $\mathcal{T} \mathcal{P}$ in Fig. 2, The effect of the product is to unfold $\mathcal{A}$ and to mark those sequences of $\mathcal{A}$ accepted by $\mathcal{T} \mathcal{P}$ in locations $\operatorname{Accept}^{\mathcal{T P}} \cdot \mathcal{T} \mathcal{P}$ is complete, thus $\operatorname{Seq}(\mathcal{P})=\operatorname{Seq}\left(\mathcal{A} \uparrow^{X_{p}^{\mathcal{T}}{ }, X_{o}^{\mathcal{T} P}}\right.$ ) (sequences of the product are sequences of $\mathcal{A}$ lifted to $\left.X^{\mathcal{T} P}\right)$, and then $\operatorname{Traces}(\mathcal{P})=\operatorname{Traces}(\mathcal{A})$, which implies that $\mathcal{P}$ and $\mathcal{A}$ define the same sets of conformant implementations. We also have $\operatorname{Seq}_{\text {Accept }^{\mathcal{P}}}(\mathcal{P})=\operatorname{Seq}\left(\mathcal{A} \uparrow^{X_{p}^{\mathcal{T} P} ; X_{o}^{\mathcal{T} P}}\right) \cap \operatorname{Seq}_{\text {Accept }^{\mathcal{T} P}}(\mathcal{T} \mathcal{P})$ which induces $\operatorname{Traces}_{\text {Accept }^{\mathcal{P}}}(\mathcal{P})=\operatorname{Traces}\left(\operatorname{Seq}(\mathcal{A}) \cap \operatorname{Seq}_{\text {Accept }^{\mathcal{T} P}}(\mathcal{T} \mathcal{P})\right)$.

Let $\operatorname{ATraces}(\mathcal{A}, \mathcal{T} \mathcal{P})=\operatorname{Traces}_{\operatorname{Accept}} \mathcal{P}(\mathcal{P})$ and $\operatorname{RTraces}(\mathcal{A}, \mathcal{T} \mathcal{P})=\operatorname{Traces}(\mathcal{A}) \backslash$ $\operatorname{pref}(\operatorname{ATraces}(\mathcal{A}, \mathcal{T} \mathcal{P}))$ where, for a set of traces $T$, pref $(T)$ denotes the set of prefixes of traces in $T$. The principle is to select traces in $\operatorname{ATraces}(\mathcal{A}, \mathcal{T} \mathcal{P})$ and try to avoid or at least detect those in $\operatorname{RTraces}(\mathcal{A}, \mathcal{T} \mathcal{P})$ as these traces cannot be prefixes of traces of sequences satisfying the test purpose. 


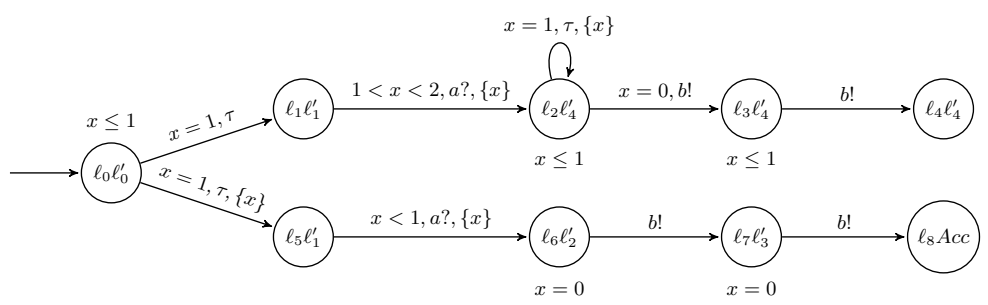

Fig. 3. Product $\mathcal{P}=\mathcal{A} \times \mathcal{T} \mathcal{P}$

Approximate determinization of $\mathcal{P}$ into $\mathcal{D} \mathcal{P}$ : If $\mathcal{P}$ is already deterministic, we simply take $\mathcal{D} \mathcal{P}=\mathcal{P}$. Otherwise, with the approximate determinization of Section 4, we can build a deterministic io-abstraction $\mathcal{D} \mathcal{P}$ of $\mathcal{P}$ with resources $\left(k, M^{\mathcal{D} P}\right)$ fixed by the user, thus $\mathcal{P} \preceq \mathcal{D P}$. $\mathcal{D} \mathcal{P}$ is equipped with the set of marked locations Accept ${ }^{\mathcal{D P}}$ consisting of locations in $L^{\mathcal{D P}}$ containing some configuration whose location is in Accept $^{\mathcal{P}}$. If the determinization is exact, we get $\operatorname{Traces}(\mathcal{D P})=\operatorname{Traces}(\mathcal{P})$ and $\operatorname{Traces}_{\mathrm{Accept}^{\mathcal{D} P}}(\mathcal{D} \mathcal{P})=\operatorname{ATraces}(\mathcal{A}, \mathcal{T} \mathcal{P})$. Fig. 4 partially represents the game $\mathcal{G}_{\mathcal{P},(1,2)}$ for the TAIO $\mathcal{P}$ of Fig. 3 where, for readability reasons, some behaviors not co-reachable from Accept $^{\mathcal{D P}}$ are omitted. $\mathcal{D} \mathcal{P}$ is simply obtained from $\mathcal{G}_{\mathcal{P},(1,2)}$ by merging transitions of Spoiler and Determinizator.

Generating $\mathcal{T C}$ from $\mathcal{D P}$ : The next step consists in building ( $\mathcal{T C}$, Verdicts) from $\mathcal{D} \mathcal{P}$, using an analysis of the co-reachability to locations Accept $^{\mathcal{D P}}$ in $\mathcal{D} \mathcal{P}$.

The test case built from $\mathcal{D} \mathcal{P}=\left(L^{\mathcal{D} P}, \ell_{0}^{\mathcal{D P}}, \Sigma_{?}^{\mathcal{D P}}, \Sigma_{!}^{\mathcal{D} P}, X_{p}^{\mathcal{D P}}, \emptyset, M^{\mathcal{D} P}, I^{\mathcal{D} P}, E^{\mathcal{D} P}\right)$ and Accept $^{\mathcal{D} P}$ is the TAIO $\mathcal{T C}=\left(L^{\mathcal{T} C}, \ell_{0}^{\mathcal{T} C}, \Sigma_{?}^{\mathcal{T} C}, \Sigma_{!}^{\mathcal{T C}}, X_{p}^{\mathcal{T} C}, \emptyset, M^{\mathcal{T} C}, I^{\mathcal{T} C}, E^{\mathcal{T} C}\right)$

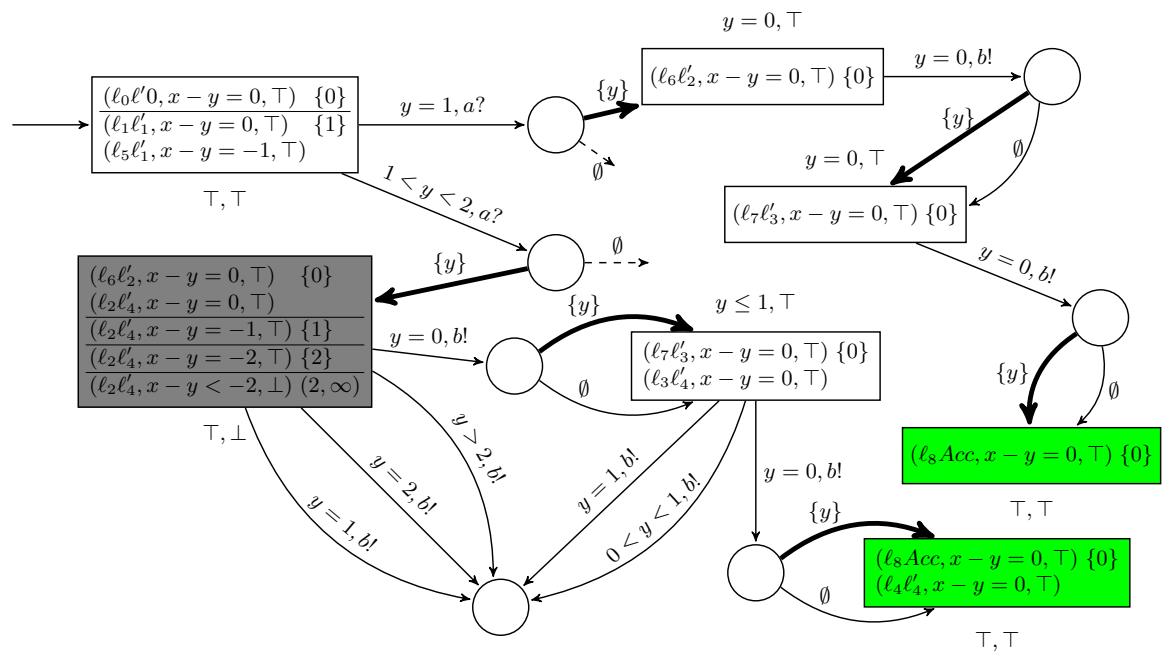

Fig. 4. Game $\mathcal{G}_{\mathcal{P},(1,2)}$ 
such that $L^{\mathcal{T} C}=L^{\mathcal{D} P} \sqcup\left\{\ell_{\text {Fail }}\right\}$ where $\ell_{\text {Fail }}$ is a new location; $\ell_{0}^{\mathcal{I C}}=\ell_{0}^{\mathcal{D P}} ; \Sigma_{?}^{\mathcal{T} C}=$ $\Sigma_{!}^{\mathcal{D} P}=\Sigma_{!}^{\mathcal{A}}$ and $\Sigma_{!}^{\mathcal{T} C}=\Sigma_{?}^{\mathcal{D} P}=\Sigma_{?}^{\mathcal{A}}$, i.e. input/output alphabets are mirrored in order to reflect the opposite role of actions in the synchronization of $\mathcal{T C}$ and $\mathcal{I}$; $X_{p}^{\mathcal{T C}}=X_{p}^{\mathcal{D P}}$ and $X_{o}^{\mathcal{T C}}=\emptyset ; M^{\mathcal{T C}}=M^{\mathcal{D} P}$; Verdicts is the partition of $S^{\mathcal{T} C}$ with Pass $=\bigcup_{\ell \in \text {Accept }^{\mathcal{D} P}}\{\ell\} \times I^{\mathcal{D} P}(\ell)$, None $=\operatorname{coreach}(\mathcal{D} \mathcal{P}$, Pass $) \backslash$ Pass, Inconc $=$ $S^{\mathcal{D} P} \backslash \operatorname{coreach}(\mathcal{D} \mathcal{P}$, Pass $)$, and Fail $=\left\{\ell_{\text {Fail }}\right\} \times \mathbb{R}_{+}^{X^{\mathcal{T} C}} \sqcup\left\{\left(\ell, \neg I^{\mathcal{D} P}(\ell)\right) \mid \ell \in L^{\mathcal{D} P}\right\}$; $I^{\mathcal{T C}}(\ell)=$ true for any $\ell \in L^{\mathcal{T} C} ; E^{\mathcal{T} C}=E_{I}^{\mathcal{D P}} \sqcup E_{\ell_{\text {Fail }}}$ where $E_{I}^{\mathcal{D P}}=\{(\ell, g \wedge$ $\left.\left.I^{\mathcal{D P}}(\ell), a, X, \ell^{\prime}\right) \mid\left(\ell, g, a, X, \ell^{\prime}\right) \in E^{\mathcal{D} P}\right\}$ and $E_{\ell_{\text {Fail }}}=\left\{\left(\ell, \bar{g}, a, X_{p}^{\mathcal{T} C}, \ell_{\text {Fail }}\right) \mid \ell \in\right.$ $\left.L^{\mathcal{D} P}, a \in \Sigma_{!}^{\mathcal{D} P}, \bar{g}=\neg \bigvee_{\left(\ell, g, a, X, \ell^{\prime}\right) \in E^{\mathcal{D} P}} g\right\}$.

The important points to understand in the construction of $\mathcal{T C}$ are the completion to Fail and the computation of Inconc. For the completion, the idea is to detect unspecified outputs and delays of $\mathcal{D} \mathcal{P}$. Outputs of $\mathcal{D} \mathcal{P}$ being inputs of $\mathcal{T C}$, in any location $\ell$, for each input $a \in \Sigma_{?}^{\mathcal{T} C}=\Sigma_{!}^{\mathcal{D P}}$, a transition leading to $\ell_{\text {Fail }}$ is added, labeled with $a$, and whose guard is the negation of the disjunction of all guards of transitions labeled by $a$ and leaving $\ell$ (thus true if no $a$-action leaves $\ell$ ). Authorized delays in $\mathcal{D} \mathcal{P}$ being defined by invariants, all states in $\left(\ell, \neg I^{\mathcal{D} P}(\ell)\right), \ell \in L^{\mathcal{D} P}$, i.e. states where the invariant runs out, are put into Fail. Moreover, in each location $\ell$, the invariant $I^{\mathcal{D} P}(\ell)$ in $\mathcal{D} \mathcal{P}$ is removed and shifted to guards of all transitions leaving $\ell$ in $\mathcal{T C}$.

The computation of Inconc is based on an analysis of the co-reachability to Pass. Inconc contains all states not co-reachable from locations in Pass. Notice that coreach $(\mathcal{D} \mathcal{P}$, Pass $)$, and thus Inconc, can be computed symbolically in the region graph of $\mathcal{D} \mathcal{P}$. Fig 5 represents the test case obtained from $\mathcal{A}$ and $\mathcal{T} \mathcal{P}$.

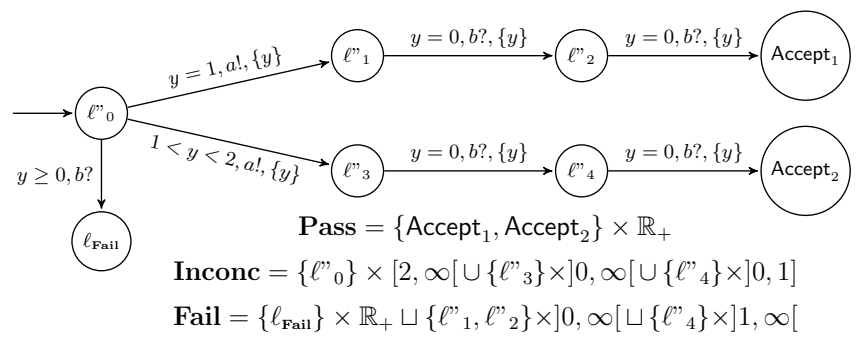

Fig. 5. Test case $\mathcal{T C}$

Test selection: So far, the construction of $\mathcal{T C}$ determines Verdicts, but does not perform any selection of behaviors. A last step consists in trying to control the behavior of $\mathcal{T C}$ in order to avoid Inconc states (thus stay in $\operatorname{pref}(\operatorname{ATraces}(\mathcal{A}, \mathcal{T} \mathcal{P}))$ ), or produce an Inconc verdict when this is impossible. To this aim, guards of transitions are refined in two complementary ways. First, transitions leaving a verdict state are useless, thus for each transition, the guard is intersected with the set of valuations associated with None in the source location. Second, transitions arriving in Inconc states and carrying inputs are also useless, thus for any transition labeled by an input, the guard is intersected with the set of valuations associated with $\operatorname{coreach}(\mathcal{D} \mathcal{P}$, Pass $)$ in the target location. 
For example in $\mathcal{T C}$ (Fig. 5), the bottom-left state of the game in Fig. 4 has been removed.

After these steps, generated test cases exhibit the following properties:

Theorem 2. Any test case $\mathcal{T C}$ built by the procedure is sound for $\mathcal{A}$. If $\mathcal{D} \mathcal{P}$ is an exact approximation of $\mathcal{P}, \mathcal{T C}$ is also strict and precise for $\mathcal{A}$ and $\mathcal{T} \mathcal{P}$.

The proof is given in the technical report 4]. Soundness comes from the construction of $E_{\text {Fail }}$ in $\mathcal{T C}$ and preservation of soundness by the approximate determinization $\mathcal{D} \mathcal{P}$ of $\mathcal{P}$ given by Corollary 1 When $\mathcal{D} \mathcal{P}$ is an exact determinization of $\mathcal{P}, \operatorname{Traces}(\mathcal{D} \mathcal{P})=\operatorname{Traces}(\mathcal{P})=\operatorname{Traces}(\mathcal{A})$. Strictness then comes from the fact that $\mathcal{D} \mathcal{P}$ and $\mathcal{A}$ have the same non-conformant traces and from the definition of $E_{\text {Fail }}$ in $\mathcal{T C}$. Precision comes from $\operatorname{Traces}_{\text {Accept }^{\mathcal{D} P}}(\mathcal{D P})=\operatorname{ATraces}(\mathcal{A}, \mathcal{T} \mathcal{P})$ and from the definition of Pass. When $\mathcal{D} \mathcal{P}$ is not exact however, there is a risk that some behaviors allowed in $\mathcal{D P}$ are not in $\mathcal{P}$, thus some non-conformant behaviors are not detected, even if they are executed by $\mathcal{T C}$. Similarly, some Pass verdicts may be produced for non-accepted or non-conformant behaviors.

Test execution. After test selection, it remains to execute test cases on a real implementation. As the test case is a TAIO, a number of decisions still need to be made at each node of the test case: (1) whether to wait for a certain delay, to receive an input or emit an output (2) which output to send, in case there is a choice. Some of these choices can be made either randomly, or according to user-defined strategies, for example by applying a technique similar to the control approach of $[8]$ whose goal is to avoid $\operatorname{RTraces}(\mathcal{A}, \mathcal{T} \mathcal{P})$.

\section{Conclusion}

In this paper, we presented a complete formalization and operations for the automatic off-line generation of test cases from non-deterministic timed automata with inputs and outputs (TAIOs). The model of TAIOs is general enough to take into account non-determinism, partial observation and urgency. One main contribution is the ability to tackle any TAIO, thanks to an original approximate determinization procedure. Another main contribution is the selection of test cases with expressive OTAIOs test purposes, able to precisely select behaviors based on clocks and actions of the specification as well as proper clocks. Test cases are generated as TAIOs using a symbolic co-reachability analysis of the observable behaviors of the specification guided by the test purpose.

Related work and discussion: As mentioned in the introduction, off-line test selection is in general limited to deterministic or determinizable timed automata, except in [14] which relies on an approximate determinization. Compared to this work, our approximate determinization is more precise (it is exact in more cases) and preserves urgency in test cases as much as possible.

In several other works [139], test purposes are used for test case selection from TAIOs. In all these works, test purposes only have proper clocks, thus 
cannot observe clocks of the specification. The advantage of our definition is its generality and a fine tuning of selection. One could argue that the cost of producing a test suite can be heavy, as for each test purpose, the whole sequence of operations, including determinization, must be done. In order to avoid this, an alternative would be to define test purposes recognizing timed traces and perform selection on the approximate determinization $\mathcal{B}$ of $\mathcal{A}$. But then, the test purpose should not use $\mathcal{A}$ 's clocks as these are lost by determinization. Then, test purposes are either defined after determinization and observe $\mathcal{B}$ 's clocks, or their expressive power is further restricted by using only proper clocks in order not to depend on $\mathcal{B}$.

Concerning test selection, in $[8$, the authors propose a game approach which effect can be understood as a way to completely avoid $\operatorname{RTraces}(\mathcal{A}, \mathcal{T} \mathcal{P})$, with the possible risk to miss some or even all traces in $\operatorname{pref}(\operatorname{ATraces}(\mathcal{A}, \mathcal{T} \mathcal{P}))$. Our selection, which allows to lose the game and produce an Inconc verdict when this happens, is both more liberal and closer to usual practice.

It should be noticed that selection by test purposes can be used for test selection with respect to coverage criteria. Those coverage criteria define a set of elements (generally syntactic ones) to be covered (e.g. locations, transitions, branches, etc). Each element can then be translated into a test purpose, the produced test suite covering the given criteria.

Acknowledgements. We wish to thank the reviewers for their helpful comments.

\section{References}

1. Alur, R., Dill, D.L.: A theory of timed automata. Theoretical Computer Science 126(2), 183-235 (1994)

2. Alur, R., Henzinger, T.A., Kupferman, O., Vardi, M.Y.: Alternating refinement relations. In: Sangiorgi, D., de Simone, R. (eds.) CONCUR 1998. LNCS, vol. 1466, pp. 163-178. Springer, Heidelberg (1998)

3. Bérard, B., Gastin, P., Petit, A.: On the power of non-observable actions in timed automata. In: Puech, C., Reischuk, R. (eds.) STACS 1996. LNCS, vol. 1046, pp. 255-268. Springer, Heidelberg (1996)

4. Bertrand, N., Jéron, T., Stainer, A., Krichen, M.: Off-line test selection with test purposes for non-deterministic timed automata. Technical Report 7501, INRIA (January 2011), http://hal.inria.fr/inria-00550923

5. Bertrand, N., Stainer, A., Jéron, T., Krichen, M.: A game approach to determinize timed automata. In: FOSSACS 2011 (to appear, 2011); Extended version as INRIA report 7381, http://hal.inria.fr/inria-00524830

6. Briones, L.B., Brinksma, E.: A test generation framework for quiescent real-time systems. In: Grabowski, J., Nielsen, B. (eds.) FATES 2004. LNCS, vol. 3395, pp. 64-78. Springer, Heidelberg (2005)

7. David, A., Larsen, K.G., Legay, A., Nyman, U., Wasowski, A.: Timed I/O automata: a complete specification theory for real-time systems. In: HSCC 2010, pp. 91-100. ACM Press, New York (2010)

8. David, A., Larsen, K.G., Li, S., Nielsen, B.: Timed testing under partial observability. In: ICST 2009, pp. 61-70. IEEE Computer Society, Los Alamitos (2009) 
9. En-Nouaary, A., Dssouli, R.: A guided method for testing timed input output automata. In: Hogrefe, D., Wiles, A. (eds.) TestCom 2003. LNCS, vol. 2644, pp. 211-225. Springer, Heidelberg (2003)

10. Finkel, O.: Undecidable problems about timed automata. In: Asarin, E., Bouyer, P. (eds.) FORMATS 2006. LNCS, vol. 4202, pp. 187-199. Springer, Heidelberg (2006)

11. Jard, C., Jéron, T.: TGV: theory, principles and algorithms. Software Tools for Technology Transfer 7(4), 297-315 (2005)

12. Khoumsi, A., Jéron, T., Marchand, H.: Test cases generation for nondeterministic real-time systems. In: Petrenko, A., Ulrich, A. (eds.) FATES 2003. LNCS, vol. 2931, pp. 131-145. Springer, Heidelberg (2004)

13. Koné, O., Castanet, R., Laurencot, P.: On the fly test generation for real time protocols. In: ICCCN 1998, pp. 378-387. IEEE, Los Alamitos (1998)

14. Krichen, M., Tripakis, S.: Conformance testing for real-time systems. Formal Methods in System Design 34(3), 238-304 (2009)

15. Nielsen, B., Skou, A.: Automated test generation from timed automata. Software Tools for Technology Transfer 5(1), 59-77 (2003)

16. Schmaltz, J., Tretmans, J.: On conformance testing for timed systems. In: Cassez, F., Jard, C. (eds.) FORMATS 2008. LNCS, vol. 5215, pp. 250-264. Springer, Heidelberg (2008)

17. Tretmans, J.: Test generation with inputs, outputs and repetitive quiescence. Software - Concepts and Tools 3, 103-120 (1996)

18. Tripakis, S.: Folk theorems on the determinization and minimization of timed automata. Information Processing Letters 99(6), 222-226 (2006) 